\title{
Acute Acalculous Cholecystitis as Initial Presentation of Amyloidosis
}

\author{
Sanjay Prakash Neupane ${ }^{a, c}$, Pratibha Sharma $^{b}$
}

\begin{abstract}
Amyloidosis is a relatively rare disease characterized by deposition of abnormal protein on any tissue or organ. It can present with a wide range of clinical manifestations depending on organ system involvement. We present here a 47-year-old Asian woman with history of hypothyroidism who came to emergency room with intermittent right upper quadrant abdominal pain for a month with worsening of symptoms for the last 3 - 4 days. Physical examination revealed mild abdomen distension, right upper quadrant tenderness, hepatomegaly and bipedal pitting edema. Her pertinent laboratory findings were serum albumin of 1.6 , total bilirubin of 3.5 , direct of 2.1 , AST/ ALT of 151/81 and alkaline phosphatase of 1,339. Her blood urea nitrogen/Cr was 20/1.6 and urinalysis was positive for proteinuria of $300 \mathrm{mg} / \mathrm{dL}$. Ultrasound (US) of gallbladder revealed hepatomegaly, thickened gallbladder wall with $4 \mathrm{~mm}$ gall bladder polyps vs. non-shadowing stones concerning for acute cholecystitis. Her viral serologies were suggestive of past hepatitis B infection. Antinuclear antibody, anti-smooth muscle antibody, anti-mitochondrial antibody, alpha- 1 antitrypsin, ceruloplasmin and iron profile were within normal limits. Liver biopsy was positive for amyloidosis. There are very few cases reported in the literature of amyloidosis initially presenting with features of acute cholecystitis and cholestatic pattern of liver function tests along with ascites. Our patient presented clinically as acute cholecystitis. The clinical dilemma was more when US commented on the findings as non-shadowing stones vs. polyps. Diffuse wall thickening of gall bladder as seen in US in this case might just be amyloid deposition rather than actual inflammation and the non-shadowing stones could as well be the amyloid deposition. Cholecystitis secondary to gallbladder localization of amyloidosis is very unusual. In conclusion, amyloidosis can rarely present as acute cholecystitis and there could be diagnostic dilemma because of inconclusive US findings.
\end{abstract}

Keywords: Amyloidosis; Cholecystitis; Gallbladder polyp; Gallstone

Manuscript accepted for publication April 29, 2015

${ }^{a}$ Internal Medicine, Maimonides Medical Center, 864 49th St, Apt B5, Brooklyn, NY, USA

${ }^{b}$ Kathmandu University School of Medical Sciences, Nepal

${ }^{\mathrm{c} C}$ Corresponding Author: Sanjay Prakash Neupane, Internal Medicine, Maimonides Medical Center, 864 49th St, Apt B5, Brooklyn, NY, USA. Current address: Internal Medicine, PeaceHealth Southwest Medical Center, 905 SE 136th Avenue, Apt B5, Vancouver, WA, USA. Email: spn894@gmail.com

doi: http://dx.doi.org/10.14740/jmc2159w

\section{Introduction}

Amyloidosis is a relatively rare disease characterized by deposition of abnormal protein on any tissue or organ [1]. There are several secondary causes for amyloidosis like chronic inflammatory process or malignancies. There is no definitive cure for amyloidosis yet. It can present with a wide range of clinical manifestations depending on organ system involvement.

\section{Case Report}

We present here a 47-year-old Asian woman with history of hypothyroidism who came to emergency room with intermittent right upper quadrant abdominal pain for a month with worsening of symptoms for the last 3 - 4 days prior to presentation. Symptoms were associated with nausea, bloating sensation, abdomen distension, early satiety and weight loss. On examination, she had mild abdomen distension, right upper quadrant tenderness, hepatomegaly and bipedal pitting edema. Her pertinent laboratory findings were serum albumin of 1.6, total bilirubin of 3.5, direct of 2.1, AST/ALT of 151/81 and alkaline phosphatase of 1,339 . Her blood urea nitrogen (BUN)/ Cr was 20/1.6 and urinalysis (UA) was positive for proteinuria of $300 \mathrm{mg} / \mathrm{dL}$. Ultrasound (US) of gallbladder revealed hepatomegaly, thickened gallbladder wall with $4 \mathrm{~mm}$ gall bladder polyps vs. non-shadowing stones concerning for acute cholecystitis. Cholestatic pattern of liver enzymes prompted us to get magnetic resonance imaging and magnetic resonance cholangiopancreatography, which confirmed the findings of US gallbladder along with ascites without any intrahepatic or extrahepatic biliary dilatation. Her viral serologies were suggestive of past hepatitis B infection. Antinuclear antibody (ANA), anti-smooth muscle antibody, anti-mitochondrial antibody, alpha-1 antitrypsin, ceruloplasmin and iron profile were within normal limits. Patient felt better with supportive treatment by day 3 of admission. Since most of the workup was unrevealing, decision was made for liver biopsy. Biopsy was positive for amyloidosis.

\section{Discussion}

Clinical presentation of amyloidosis depends on the type of protein, tissue distribution and the amount of amyloid depo- 
sition [2]. Some clinical and laboratory features that suggest amyloidosis include waxy skin and easy bruising, enlarged tongue, symptoms and signs of heart failure, cardiac conduction abnormalities, hepatomegaly, proteinuria, peripheral and/or autonomic neuropathy and impaired coagulation [3]. Proteinuria leading to nephrotic syndrome and renal insufficiency is the earliest and most frequent clinical manifestation that should raise suspicion of AA amyloidosis [2]. There are very few cases reported in the literature of amyloidosis initially presenting with features of acute cholecystitis and cholestatic pattern of liver function tests along with ascites. Our patient presented clinically as acute cholecystitis. The clinical dilemma was more when US commented on the findings as non-shadowing stones vs. polyps. Diffuse wall thickening of gall bladder as seen in US in this case might just be amyloid deposition rather than actual inflammation and the non-shadowing stones could as well be the amyloid deposition. Cholecystitis secondary to gallbladder localization of amyloidosis is very unusual. Generally amyloidosis affects the epithelium of intrahepatic bile duct without reaching canaliculi and acini of glandular peribiliares and amount of bile deposits correlates with the extent of amyloidosis in liver parenchyma [4].

\section{Conclusion}

Amyloidosis can rarely present as acute cholecystitis and there could be diagnostic dilemma because of inconclusive US findings.

\section{Source of Support}

None.

\section{Conflicts of Interest}

None.

\section{Grant Support}

None.

\section{References}

1. Culafic D, Perisic M, Boricic I, Culafic-Vojinovic V, Vukcevic M. Primary amyloidosis presenting with cholestasis and hyperkinetic portal hypertension. J Gastrointestin Liver Dis. 2007;16(2):201-204.

2. Lachmann HJ, Goodman HJ, Gilbertson JA, Gallimore JR, Sabin CA, Gillmore JD, Hawkins PN. Natural history and outcome in systemic AA amyloidosis. N Engl J Med. 2007;356(23):2361-2371.

3. Pepys MB. Amyloidosis. Annu Rev Med. 2006;57:223241.

4. Kwon AH, Tsuji K, Yamada H, Okazaki K, Sakaida N. Amyloidosis of the gallbladder mimicking gallbladder cancer. J Gastroenterol. 2007;42(3):261-264. 